\title{
聚二甲基硅氧烷封装石墨烯基柔性红外探测器的制备及其应用
}

\author{
赵雅婧 $\dagger, a, c$ 谢亮 ${ }^{\dagger,} a, b$ 马兰超 ${ }^{b}$ 贺军辉*, $a$ \\ ( ${ }^{a}$ 中国科学院理化技术研究所 微纳材料与技术研究中心 功能纳米材料实验室 北京 100190) \\ ( ${ }^{b}$ 北京石油化工学院 材料科学与工程学院 北京 102617) \\ ( ${ }^{c}$ 中国科学院大学 北京 100049)
}

\begin{abstract}
摘要 采用热还原法在不同退火温度(从 100 到 $1200{ }^{\circ} \mathrm{C}$ )下制备了一系列还原氧化石墨烯 $(\mathrm{rGO})$ 薄膜, 并通过 $\mathrm{X}$ 射线粉 末衍射(XRD)、傅里叶变换红外光谱(FTIR)、拉曼光谱、四探针电阻仪和扫描电镜(SEM)对其进行了表征和分析. 随后 利用旋涂封装法制备了聚二甲基硅氧烷(PDMS)封装的石墨烯基柔性红外探测器(P-rGO-P), 并成功用于红外激光、人体 红外辐射、弯曲变化和压力探测. 实验结果表明, 采用不同温度还原的 rGO 薄膜封装得到的 P-rGO-P 柔性红外探测器 在近红外(1064 nm)激光照射下均有响应, 其中最高的光响应可达 $2.78 \mathrm{~mA} / \mathrm{W}$. 此外, P-rGO-P 柔性探测器对人体红外辐 射和弯曲变化也具有快速、灵敏的响应, 并且经过反复弯折仍能保持完整性与响应能力. 关键词 氧化还原石墨烯; 柔性; 光电转换; 红外探测器; 压力探测器
\end{abstract}

\section{Preparation and Application of Polydimethylsiloxane Encapsulated Graphene-based Flexible Infrared Detector}

\author{
Zhao, Yajing ${ }^{\dagger, a, c} \quad$ Xie, Liang ${ }^{\dagger}, a, b \quad$ Ma, Lanchao $^{b} \quad$ He, Junhui*,a \\ ( ${ }^{a}$ Functional Nanomaterials Laboratory, Center for Micro/nanomaterials and Technology, Technical Institute of Physics and \\ Chemistry, Chinese Academy of Sciences, Beijing 100190, China) \\ ( ${ }^{b}$ College of Materials Science \& Engineering, Beijing Institute of Petrochemical Technology, Beijing 102617, China) \\ ( ${ }^{c}$ University of Chinese Academy of Sciences, Beijing 100049, China)
}

\begin{abstract}
In this paper, we prepared reduced graphene oxide (rGO) films by first drop-casting graphene oxide (GO)/ethanol dispersion on top of silicon nanowires array, followed by thermal reduction in $95 \% \mathrm{Ar}-5 \% \mathrm{H}_{2}$ (volume ratio) atmosphere. A series of rGO thin films were prepared by thermal reduction at different annealing temperatures ranging from $100{ }^{\circ} \mathrm{C}$ to $1200{ }^{\circ} \mathrm{C}$, and characterized by X-ray diffraction (XRD), Fourier transform infrared spectroscopy (FTIR), Raman spectroscopy, four-probe square resistance tester and scanning electron microscopy (SEM). The experimental results indicate that reduction of oxygen-containing groups, dehydrogenation of $\mathrm{C}-\mathrm{H}$ groups and reconstruction of $\mathrm{C}=\mathrm{C}$ skeleton occurred significantly on the GO plane. Compared with the insulating GO film, the resistance of rGO thin films decreases greatly, and the sheet resistance of rGO films shows a decreasing trend with increase of reduction temperature. Then, flexible polydimethylsiloxane (PDMS) encapsulated graphene-based devices (P-rGO-P) were fabricated by spin-coating PDMS on the surface of obtained rGO films with evaporated $\mathrm{Au}$ interdigital electrodes. The flexible devices maintained the integrity of the rGO films while providing self-supporting characteristics. The rGO film in the device had a clear layered structure, and a certain movable space between the upper and lower PDMS layers. This sandwich structure ensures that when the P-rGO-P flexible detector is bent and squeezed, the rGO film has sufficient buffer space, and would not be subjected to excessive stress arising from adhesion to PDMS. In short, the sandwich structure endows the originally fragile device with excellent flexibility. The P-rGO-P detector was successfully applied to detecting infrared laser irradiation, human body infrared radiation, bending motions and pressure changes. The experimental results showed that the flexible encapsulated P-rGO-P infrared detectors derived from the rGO thin films reduced at varied temperatures all had response to near-infrared (1064 $\mathrm{nm})$ laser irradiation, and the maximum response reached up to $2.78 \mathrm{~mA} / \mathrm{W}$. In addition, the P-rGO-P flexible detector also demonstrated fast and sensitive response to human body infrared radiation and bending changes, and could maintain its integrity and responsiveness after repeated bending.
\end{abstract}

Keywords reduced graphene oxide; flexibility; photoelectric conversion; infrared detector; strain detector

* E-mail: jhhe@mail.ipc.ac.cn; Tel.: 010-82543535; Fax: 010-82543535

$\uparrow$ These authors contributed equally to this work.

Received October 22, 2019; published January 10, 2020.

Supporting information for this article is available free of charge via the Internet at http://sioc-journal.cn

Project supported by the National Natural Science Foundation of China (No. 21571182), the National Key Research and Development Program of China (No. 2017YFA0207102), the Science and Technology Commission of Beijing Municipality (No. Z151100003315018) and the Beijing "Practical Training Program".

项目受国家自然科学基金(No. 21571182)、国家重点研究开发项目(No. 2017YFA0207102)、北京市先导与优势材料创新项目(No. Z151100003315018) 和北京 “实培计划” 资助. 


\section{1 引言}

近年来, 随着红外光电探测器在医学诊断、光通信、 资源勘探、军用侦测等 ${ }^{[177}$ 领域中展现出越来越重要的 应用潜力, 其相关技术得到了快速发展. 但目前高响应 率、高探测率的红外探测材料, 如 $\mathrm{III} \sim \mathrm{V}$ 族的二元系半 导体 ${ }^{[8]}$, 仅能够吸收能量与其带隙相匹配的光子, 且需 要复杂的制造工艺和高成本的低噪音运行冷却机制. 此 外, 现代社会对各种电子器件小型化、便携性、柔性、 低成本和宽波长响应等性能的需求也在不断增长 ${ }^{[9]}$, 而 传统的半导体材料一般不具备柔性, 在拉伸过程中易于 损坏或者失效. 于是, 探索新的自身具有柔性的红外探 测材料吸引了科学家和工程师的关注, 如碳材料和有机 材料.

自 2004 Geim 课题组首次利用胶带剥离方法从石墨 上成功剥离、观察到石墨烯(Graphene), 并揭示了其 $\mathrm{sp}^{2}$ 杂化碳原子形成的六方蜂巢二维晶体结构 ${ }^{[10]}$ 以来, 石 墨烯就引起了广泛的关注与研究. 石墨烯具有从紫外、 可见到红外甚至太赫兹波段的宽光谱吸收特性, 室温下 有着超高的电子迁移率、超大比表面积、良好的环境稳 定性等优良性能 ${ }^{[10 ~ 12]}$, 使其在超宽谱、超快、非制冷、 大面阵、柔性和长寿命光电探测器方面极具潜力.

通常, 在研究颇广的基于石墨烯材料并利用光电门 效应工作的混合光晶体管中, 石墨烯仅作为电荷传导通 道, 起有效地传输光生电子或空穴的作用. 研究人员需

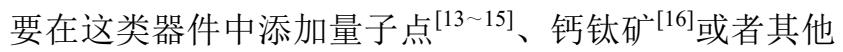
二维半导体 ${ }^{[17,18]}$ 来吸收入射光并产生电子空穴对. 为了 解决石墨烯因零带隙特征引起的低响应率和低光电增 益问题, 研究人员选择了不破坏石墨烯本身结构, 改变 其对称性或是通过剪切形成纳米带等方法来打开石墨 烯带隙 ${ }^{[19]}$. 另有研究人员通过相对简单过程, 制备了成 本低廉、具有受还原程度调控带隙的还原氧化石墨烯 (rGO) 材料 ${ }^{[20]}$.

本课题组 ${ }^{[21 ~ 23]}$ 最近的研究结果表明, 在预处理后 形成纳米线阵列的基底上, 逐层滴涂氧化石墨烯 (GO) 的乙醇分散液, 干燥后经过热处理可以揭下具有优异导 电性的 $\mathrm{rGO}$ 薄膜. 采用这种薄膜制备的自支撑光电探测 器在近红外波段(1064 nm) 展现出了优秀的响应率, 且 薄膜自身具有一定的柔性, 但受外力极易遭到破坏, 无 法适应柔性器件对于弯曲和拉伸的需求.

本工作在此研究基础上, 利用聚二甲基硅氧烷 (polydimethylsiloxane, PDMS) 封装的方法, 简单、高效地 制备了一种具有三明治结构的柔性石墨烯基红外探测 器(P-rGO-P 柔性红外探测器). PDMS 具有很好的柔韧 性、拉伸性、耐腐蚀性、易操作性 ${ }^{[24]}$. 这种基于 PDMS 的三明治包夹结构使得器件的柔性有了极大的提高, 弯 折角度可达 $180^{\circ}$. 进一步针对在近红外波段 (1064 nm) 有较好响应率的 $\mathrm{rGO}$ (200 和 $1000{ }^{\circ} \mathrm{C}$ 下热还原), 研究 了邻近还原温度 $\left(100\right.$ 到 $300{ }^{\circ} \mathrm{C}$ 和 800 到 $\left.1200{ }^{\circ} \mathrm{C}\right)$ 对 $\mathrm{rGO}$
的影响，以及制得的柔性红外探测器在近红外波段和人 体红外波段 $(9 \sim 10 \mu \mathrm{m})$ 的光响应性, 并探索了 P-rGO-P 柔性红外探测器的多功能性.

\section{2 结果与讨论}

\section{1 制备流程}

P-rGO-P 柔性红外探测器件的制备与组装流程如图 1 所示. 首先, 在硅纳米线上滴涂 $\mathrm{GO}$ 分散液, 烘干后在 $95 \% \mathrm{Ar}-5 \% \mathrm{H}_{2}$ (体积比)气氛下, 经由高温还原制备 $\mathrm{rGO}$ 薄膜 ${ }^{[23]}$. 剥离 $\mathrm{rGO}$ 薄膜, 在其表面真空蒸镀 $\mathrm{Au}$ 叉指电 极. 然后将该薄膜粘在自制的 PDMS 基底上, 并在电极 上粘接 $\mathrm{Cu}$ 导线, 待干燥后旋涂 PDMS 进行封装, 最终 烘干得到 P-rGO-P 柔性红外探测器件.

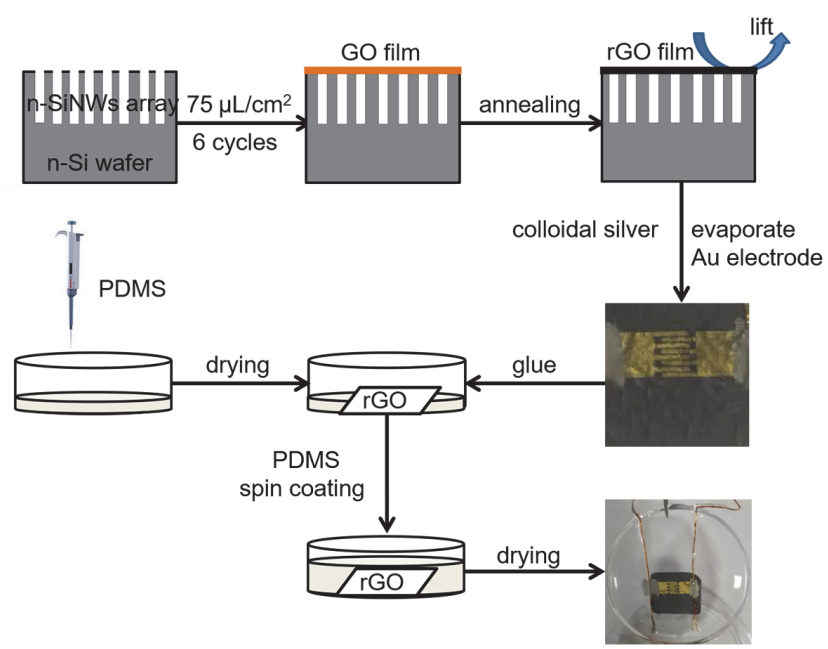

图 1 P-rGO-P 柔性红外探测器件的制备与组装流程示意图

Figure 1 Schematic illustration of the preparation strategy of P-rGO-P flexible infrared detector

\section{2 还原结果}

如图 2 所示, 测试所得 XRD 图谱峰位符合 $\mathrm{GO}$ 特征 峰峰位 $\left(2 \theta=11^{\circ}\right)$ 与 $\mathrm{rGO}$ 特征峰峰位 $\left(2 \theta=24^{\circ}\right)^{[25]}$.

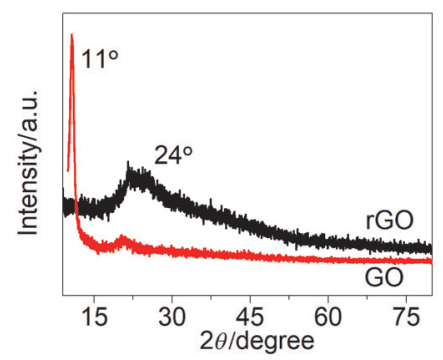

图 $2 \mathrm{GO}$ 与 $\mathrm{rGO}$ 的 XRD 谱图

Figure 2 XRD spectra of GO and rGO

层间距可通过布拉格公式计算

$2 d \sin \theta=n \lambda$

其中 $d$ 为层间距, $\theta$ 为入射 $\mathrm{X}$ 射线与相应晶面的夹角, $n$ 为衍射级数, $\lambda$ 为 $\mathrm{X}$ 射线波长, 本文测试所用 $\mathrm{X}$ 射线为 
$\mathrm{Cu}$ 靶 $\mathrm{K} \alpha$ 射线 $(\lambda=0.15406 \mathrm{~nm})$.

由公式(1)计算得到 $\mathrm{GO}$ 的层间距为 $0.80 \mathrm{~nm}, \mathrm{rGO}$ 的 层间距为 $0.37 \mathrm{~nm}$. 计算结果表明 $\mathrm{rGO}$ 层间距减小, 这 是 $\mathrm{GO}$ 得到有效还原后所含含氧官能团减少所致.

从 $\mathrm{GO}$ 薄膜与各个温度下还原所得的 $\mathrm{rGO}$ 薄膜的 FTIR 光谱(图 3)可以清晰地观察到, 相比于 GO 薄膜, $\mathrm{rGO}$ 在还原后所有含氧官能团对应峰强都有了明显的 减小, 如中心位置在 $3400 \mathrm{~cm}^{-1}$ 的 $-\mathrm{OH}$ 宽峰, 以及峰位 在 $1726 \mathrm{~cm}^{-1}$ 的 $\mathrm{C}=\mathrm{O}$ 官能团和峰位在 $1219 \mathrm{~cm}^{-1}$ 的 C-O-C 官能团. 此外, GO 的红外光谱在 $2851 \sim 2926$ $\mathrm{cm}^{-1}$ 处可以观测到对应于 $\mathrm{C}-\mathrm{H}$ 伸缩振动的微弱起伏, 而 $1516 \mathrm{~cm}^{-1}$ 处对应芳环 $\mathrm{C}=\mathrm{C}$ 伸缩振动的峰强相较 $\mathrm{rGO}$ 的更弱, 这印证了还原过程中发生了 $\mathrm{C}-\mathrm{H}$ 基团的脱 氢和 $\mathrm{C}=\mathrm{C}$ 骨架的重建.

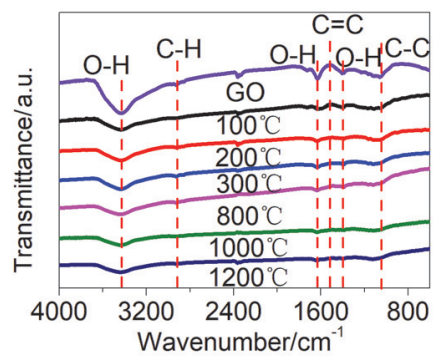

图 $3 \mathrm{GO}$ 薄膜与不同温度 $\left(100,200,300,800,1000,1200{ }^{\circ} \mathrm{C}\right)$ 下还原 所得的 $\mathrm{rGO}$ 薄膜的 FTIR 光谱图

Figure 3 FTIR spectra of GO film and rGO films reduced at different temperatures $\left(100,200,300,800,1000,1200{ }^{\circ} \mathrm{C}\right)$

为进一步证明 $\mathrm{rGO}$ 得到了有效还原, 对 $\mathrm{GO}$ 和各个 温度还原所得 $\mathrm{rGO}$ 薄膜进行了 Raman 光谱测试, 结果 如图 4 所示. Raman 光谱表明 GO 和 rGO 薄膜在 1350 $\mathrm{cm}^{-1}$ 和 $1589 \mathrm{~cm}^{-1}$ 处均有分别归属 $\mathrm{D}$ 峰和 $\mathrm{G}$ 峰的突出 特征峰, 几乎所有 $\mathrm{rGO}$ 的两峰强度比值 $\left(I_{\mathrm{D}} / I_{\mathrm{G}}\right)$ 相较 $\mathrm{GO}$ 的都有明显下降, 这证明 $\mathrm{rGO}$ 结构的有序性都有极大程 度的提高, 其中 $1000{ }^{\circ} \mathrm{C}$ 下还原所得 $\mathrm{rGO}$ 的 $I_{\mathrm{D}} / I_{\mathrm{G}}$ 值 (1.228)略高于 $\mathrm{GO}$ 的(1.038), 这可能是由于在 $1000{ }^{\circ} \mathrm{C}$ 下还原制备的 rGO 拥有更多较小的 $\mathrm{sp}^{2}$ 区域 ${ }^{[25]}$. 同时, $\mathrm{rGO}$ 薄膜的拉曼峰相较 GO 薄膜的更为狭窄, 且都拥有 更清晰的 $2 \mathrm{D}$ 峰, 证明热还原后的 $\mathrm{rGO}$ 拥有高结晶度,
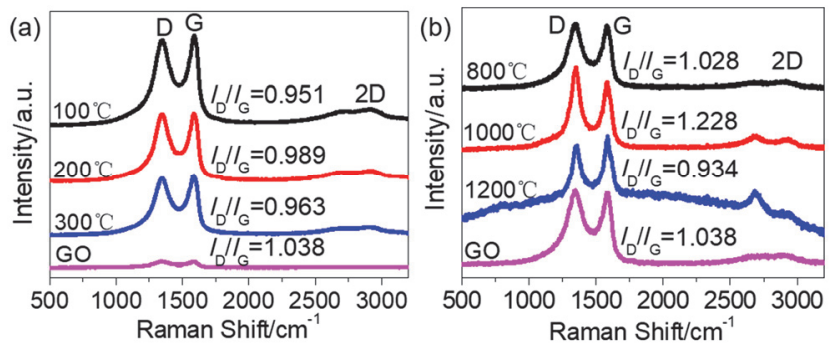

图 4 (a) GO 和分别在 $100,200,300{ }^{\circ} \mathrm{C}$ 下还原所得 $\mathrm{rGO}$ 的拉曼光谱 图; (b) GO 和分别在 $800,1000,1200{ }^{\circ} \mathrm{C}$ 下还原所得 $\mathrm{rGO}$ 的拉曼光谱图 Figure 4 Raman spectra of (a) GO and rGO reduced at 100, 200, $300{ }^{\circ} \mathrm{C}$, respectively; (b) GO and rGO reduced at $800,1000,1200{ }^{\circ} \mathrm{C}$, respectively
且形成了更加有序的石墨烯化的结构.

采用四探针台对各个温度还原所得 $\mathrm{rGO}$ 薄膜的方 块电阻进行测试, 方块电阻与还原温度的关系(图 5)表 明, 相较绝缘的 GO 薄膜, rGO 的电阻有了大幅度的降 低, 且 $\mathrm{rGO}$ 薄膜的方块电阻呈现出随着还原温度的升高 而降低的变化趋势. 当还原温度从 $200{ }^{\circ} \mathrm{C}$ 增加到 $300{ }^{\circ} \mathrm{C}$ 时, 薄膜的方块电阻从 $355.7 \Omega / \square$ 迅速下降到 $154.6 \Omega / \square$, 甚至可在 $1200{ }^{\circ} \mathrm{C}$ 时下降到极低的 $13 \Omega / \square$, 说明 $\mathrm{rGO}$ 薄膜的导电性能得到了显著改善. 由于 $100{ }^{\circ} \mathrm{C}$ 下 $\mathrm{rO}$ 的还原程度过低, 未列出其方块电阻数 据.

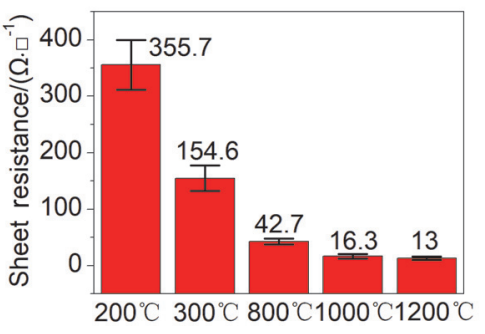

图 $5 \mathrm{rGO}$ 薄膜的方块电阻与还原温度的关系图

Figure 5 The sheet resistance as a function of thermal reduction temperature for $\mathrm{rGO}$

\section{3 器件性能}

图 6 是分别在 $100,200,300,800,1000,1200{ }^{\circ} \mathrm{C}$ 下 还原所得 rGO 薄膜的 SEM 顶视图像. 如图所示, $\mathrm{rGO}$ 薄 膜表面大致是光滑的，但由于石墨烯的天然易折叠性， 表面观察到一些褶皱. 此外, 从 rGO 薄膜宏观照片插图 可见, rGO 表面形貌均一并泛有明显的金属光泽.
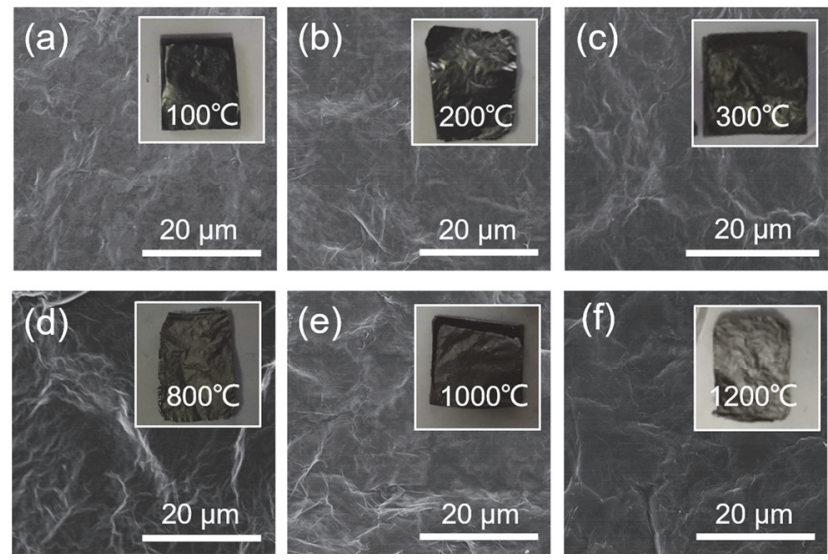

图 $6100,200,300,800,1000,1200{ }^{\circ} \mathrm{C}$ 下还原所得 $\mathrm{rGO}$ 的扫描电镜图 像. 插图为薄膜宏观照片

Figure 6 SEM images of rGO thin films annealed at 100, 200, 300, 800, $1000,1200{ }^{\circ} \mathrm{C}$, respectively. The insets are photographs of rGO thin films

由各温度下还原制备的 rGO 所组装的 P-rGO-P 柔 性器件如图 7a 所示, 从图中可以看出, 经过封装的光电 探测器在保持了 rGO 薄膜完整性的同时, 具备了柔性器 


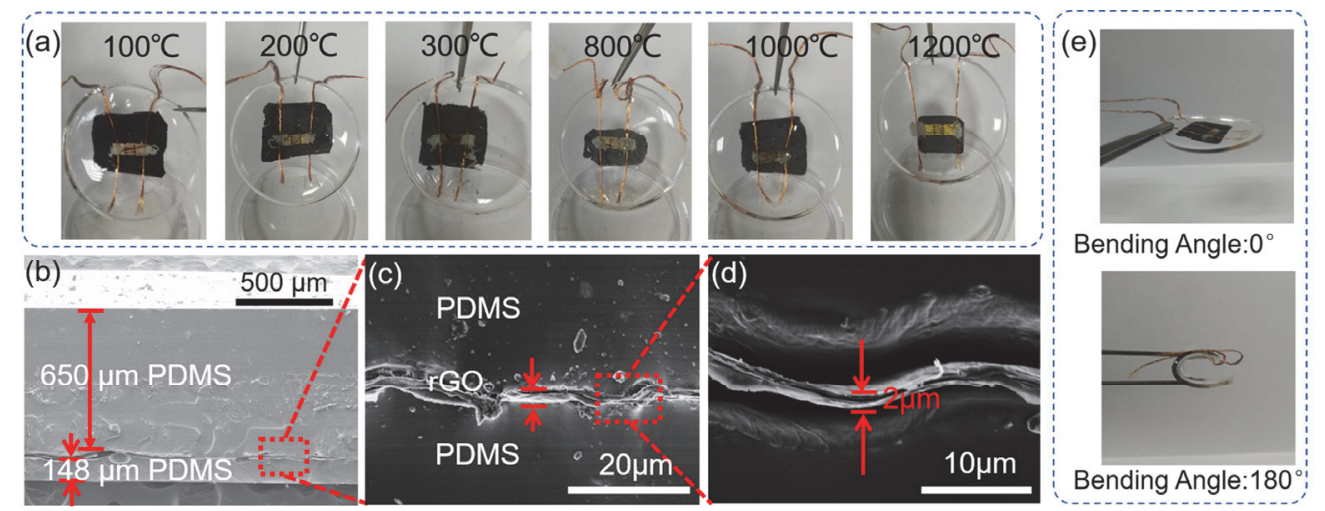

图 7 (a)由 $100,200,300,800,1000,1200{ }^{\circ} \mathrm{C}$ 下还原所得 $\mathrm{rGO}$ 制备的 P-rGO-P 器件; (b d) 器件截面扫描电镜图像; (e) P-rGO-P 器件柔性测试照片 Figure 7 (a) Photographs of P-rGO-P detectors prepared by reduced rGO at 100, 200, 300, 800, 1000, $1200{ }^{\circ} \mathrm{C}$, respectively; (b $\left.\sim \mathrm{d}\right)$ cross-sectional SEM images of the P-rGO-P detector; (e) flexibility test photographs of P-rGO-P detectors

件需要的独立自支撑性. 图 7b 7d 是其截面 SEM 图像. 从截面图中可知, rGO 薄膜具有清晰的层状结构, 整个 柔性器件的厚度约为 $800 \mu \mathrm{m}$, 其中上层 PDMS 厚度为 $650 \mu \mathrm{m}$, 下层 PDMS 厚度为 $148 \mu \mathrm{m}, \mathrm{rGO}$ 薄膜厚度仅为 $2 \mu \mathrm{m}$, 并且与上下方的 PDMS 都留有一定可活动的空 隙, 这保证了 P-rGO-P 柔性器件在受到弯曲作用力时, $\mathrm{rGO}$ 薄膜不会因 PDMS 层的拉扯而破损. 从图 7e 的弯 曲测试可见, PDMS 封装使得原本脆弱的器件拥有了优 秀的柔性, 可以轻松弯折至 $180^{\circ}$, 不再极易破损.

为考察 P-rGO-P 柔性器件在红外波段的光响应能 力, 作为对照, 我们也使用分别在 $100,200,300,800$ 1000 和 $1200{ }^{\circ} \mathrm{C}$ 下还原所得的 $\mathrm{rGO}$ 薄膜, 通过真空蒸镀 金电极, 将镀有电极的薄膜悬空固定在玻璃支架基底 上, 粘结铜导线, 从而制备了一批无 PDMS 封装的自支 撑 $\mathrm{rGO}$ 光电探测器 (器件示意图见 Supporting Information, SI, 图 S1), 之后对两组器件在相同条件下进行 了光电测试. 所有的测试均是在室温与空气下进行的, 测试中所用的入射激光为光斑大小为 $3 \mathrm{~mm}$, 波长为 $1064 \mathrm{~nm}$ 的红外激光. 将测试样品固定在样品台上, 通 过调节样品台在垂直和水平方向上移动, 使入射激光的 光斑能刚好照射在器件镀有叉指电极的中心位置. 利用 光电快门来控制光电探测器是否受到光照, 并将所得测 试结果中的光照电流 $\left(I_{\mathrm{light}}\right)$ 和暗黑电流 $\left(I_{\mathrm{dark}}\right)$ 分别定义为 光电探测器在有光照和无光照时的稳态电流, 二者的差 值 $\left(I_{\text {light }}-I_{\text {dark }}\right)$ 则被定义为光电探测器的光电流 $\left(I_{\mathrm{ph}}, I_{\mathrm{ph}}=\right.$ $\left.I_{\text {light }}-I_{\text {dark }}\right)$.

图 $8 \mathrm{a} \sim 8 \mathrm{f}$ 分别为没有 $P D M S$ 封装的自支撑 $\mathrm{rGO}$ 光 电探测器在功率 $64.8 \mathrm{~mW}$, 波长 $1064 \mathrm{~nm}$ 的红外激光照 射下, 偏置电压为 $-1 \sim 1 \mathrm{~V}$ 时进行光电测试得到的电 流-电压 $(I-V)$ 曲线. 如图所示, 红色曲线代表 $I_{\text {light }}$ 随偏置 电压变化的结果, 黑色曲线代表 $I_{\mathrm{dark}}$ 随偏置电压变化的 结果. 在 $I-V$ 曲线的放大图中, 若红色曲线在黑色曲线 上方, 证明 $I_{\mathrm{ph}}$ 值大于 0 , 反之, 则证明 $I_{\mathrm{ph}}$ 值小于 0 . 由图 可知, 基于 100 和 $200{ }^{\circ} \mathrm{C}$ 热还原所得 $\mathrm{rGO}$ 薄膜的光电探
测器, 其 $I-V$ 曲线的放大图中红色曲线位于黑色曲线上 方, 而基于 $300,800,1000$ 和 $1200{ }^{\circ} \mathrm{C}$ 热还原所得 $\mathrm{rGO}$ 薄 膜的光电探测器的 $I-V$ 曲线放大图中红色曲线位于黑色 曲线下方, 这表明随着还原温度的增加, 光电探测器的 $I_{\mathrm{ph}}$ 值从正值 $\left(100\right.$ 和 $\left.200{ }^{\circ} \mathrm{C}\right)$ 变为负值 $(300,800,1000$ 和 $\left.1200{ }^{\circ} \mathrm{C}\right)$. 这一现象在红外激光 $(1064 \mathrm{~nm})$ 照射下所得的 电流-时间关系 $(I-t)$ 曲线(图 9)中也可以清晰地观察到.

如图 9 所示, 基于 100 和 $200{ }^{\circ} \mathrm{C}$ 热还原所得 $\mathrm{rGO}$ 薄 膜的光电探测器, 当激光照射时 (ON), 其电流立刻快速 增大, 然后达到稳定. 而基于 $300,800,1000$ 和 $1200{ }^{\circ} \mathrm{C}$ 热还原所得 $\mathrm{rGO}$ 薄膜的光电探测器, 当激光照射时, 电 流会先快速减小, 然后达到稳定. 这可能是因为: 一方 面, $\mathrm{rGO}$ 表面上蒸镀的金电极作为一种金属薄膜在光照 下, 产生了光诱导表面等离子极化子, 为电子传输提供 了另一个散射通道, 并最终导致其呈现出显著的负光电 导率 ${ }^{[26,27]}$. 另一方面, rGO 具有由入射光子和伴随入射 光子的热效应引起的正光响应性 ${ }^{[28]}$. 在热还原所得的 $\mathrm{rGO}$ 薄膜中仍然有含氧官能团残余, 且随着还原温度的 升高(从 100 到 $1200{ }^{\circ} \mathrm{C}$ ), rGO 薄膜中碳氧含量比会逐渐 升高 ${ }^{[21]}$, 即含氧官能团的含量会随着还原温度的升高 而降低. 而 rGO 的正响应会受到 $\mathrm{rGO}$ 还原程度的影响, 因为 $\mathrm{rGO}$ 中的含氧缺陷可以捕获光生电子, 与之对应的 光生空穴就得以继续在外电路中循环, 提高了光生载流 子的寿命和载流子的迁移率 ${ }^{[29]}$, 从而提高外部量子效 率. 此外, 含氧官能团的存在也导致了电子传导的变程 跳跃电导(variable range hopping conductivity, VRH)传输 机制 ${ }^{[30]}$. 如上所述, 随还原温度升高, rGO 薄膜还原程 度提高, 对光生电子的捕获能力降低, 于是在外电路中 循环的光生空穴数量也随 $\mathrm{rGO}$ 还原程度的提高而降低, 导致 $\mathrm{rGO}$ 的正光响应绝对值减小, 最终在与金电极引起 的负光响应的竞争中影响到 $I_{\mathrm{ph}}$ 的正负. 例如, $\mathrm{rGO}$ 还原 温度较低的情况下, $\mathrm{rGO}$ 中氧官能团的含量较高, 正光 响应高于金叉指电极的负光电导率绝对值, 观察到的 $I_{\mathrm{ph}}$ 就大于 $0^{[21]}$. 

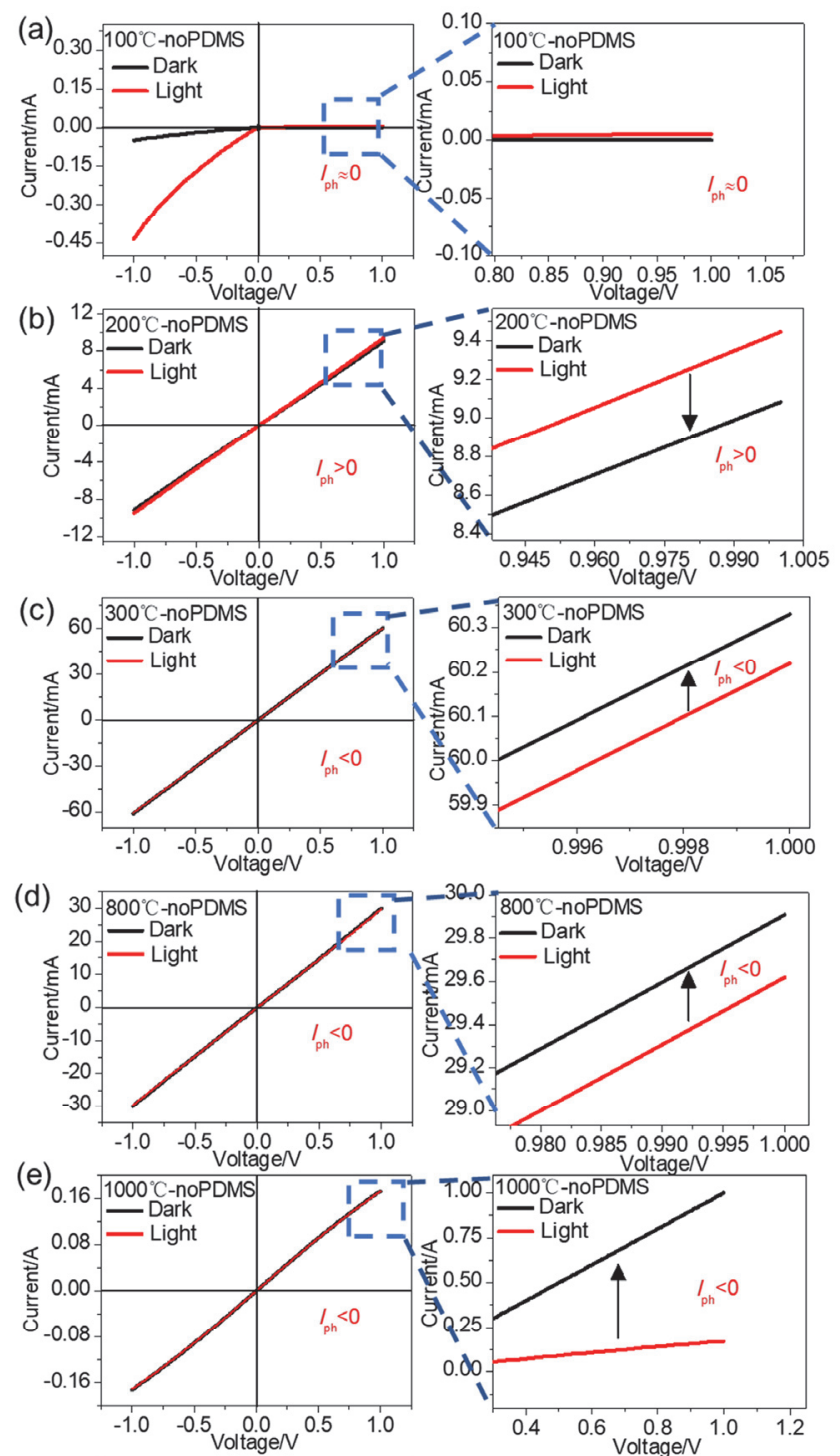

$0.980 \quad 0.985 \quad 0.990 \quad 0.9951 .000$
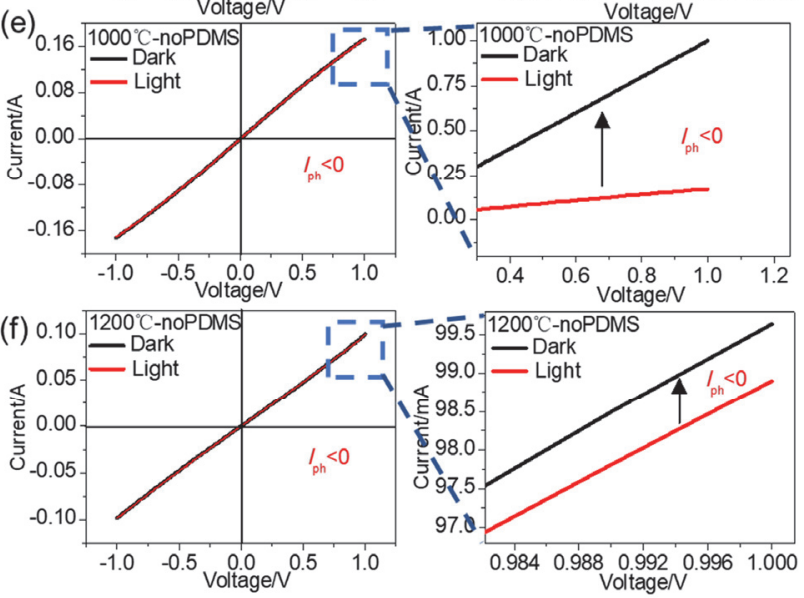

图 8 分别在无光照和 $1064 \mathrm{~nm}$ 激光照射下测试的 $I-V$ 曲线, 测试基 于 $100,200,300,800,1000,1200{ }^{\circ} \mathrm{C}$ 下热还原 $\mathrm{rGO}$ 薄膜制备的没有 PDMS 封装的光电探测器(红外激光波长: $1064 \mathrm{~nm}$, 功率: $64.8 \mathrm{~mW}$, 偏置电压: $-1 \sim 1 \mathrm{~V}$ )

Figure $8 I-V$ curves measured in dark and upon $1064 \mathrm{~nm}$ laser illumination, respectively, of unencapsulated rGO detectors based on rGO thin films annealed at $100,200,300,800,1000,1200{ }^{\circ} \mathrm{C}$ (laser wavelength: $1064 \mathrm{~nm}$, power: $64.8 \mathrm{~mW}$, bias voltage: $-1 \sim 1 \mathrm{~V}$ )

P-rGO-P 光电探测器的电流-电压 $(I-V)$ 曲线在功率 $64.8 \mathrm{~mW}$, 波长 $1064 \mathrm{~nm}$ 的红外激光照射下, 偏置电压 为 $-1 \sim 1 \mathrm{~V}$ 时进行光电测试得到, 如图 10a-10f 所示. 由图可知，基于 $100,200,300$ 和 $800{ }^{\circ} \mathrm{C}$ 热还原所得 $\mathrm{rGO}$ 薄膜的光电探测器, 其 $I-V$ 曲线的放大图中红色曲线位
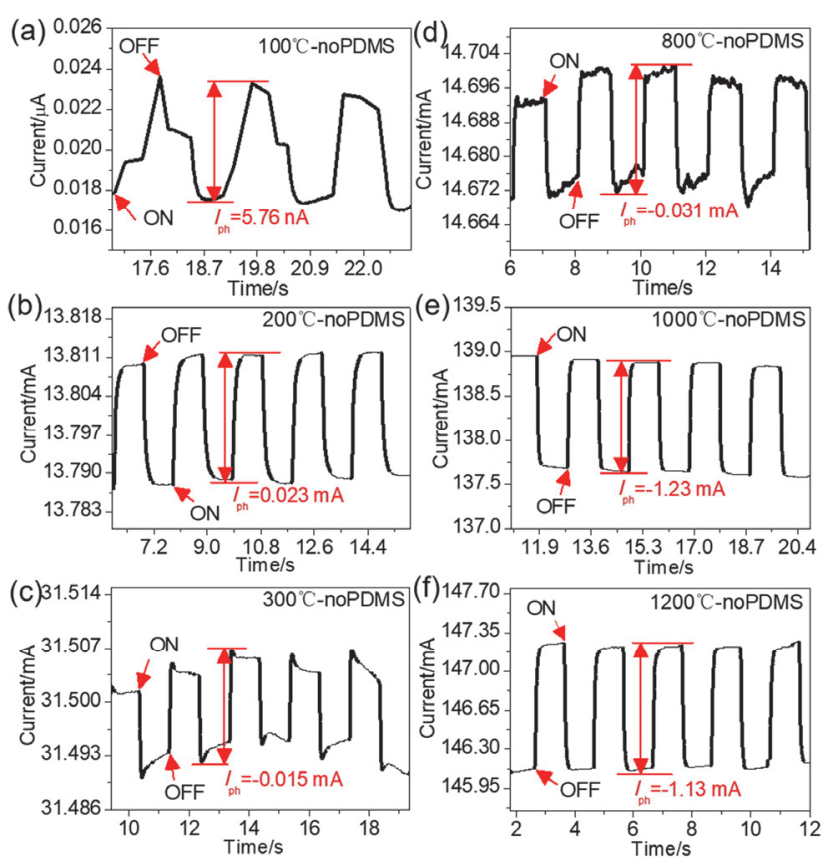

图 9 由 $100,200,300,800,1000$ 和 $1200{ }^{\circ} \mathrm{C}$ 下还原所得的没有 PDMS 封装的自支撑 $\mathrm{rGO}$ 光电探测器的电流-时间曲线(红外激光波长: 1064 $\mathrm{nm}$, 功率: $64.8 \mathrm{~mW}$, 偏置电压: $0.5 \mathrm{~V}$ )

Figure $9 I-t$ curves of unencapsulated rGO detectors based on rGO thin films annealed at $100,200,300,800,1000$ and $1200{ }^{\circ} \mathrm{C}$, respectively (laser wavelength: $1064 \mathrm{~nm}$, power: $64.8 \mathrm{~mW}$, bias voltage: $0.5 \mathrm{~V}$ )

于黑色曲线上方, 即 $I_{\mathrm{ph}}>0$. 而基于 $1000{ }^{\circ} \mathrm{C}$ 和 $1200{ }^{\circ} \mathrm{C}$ 热还原所得 $\mathrm{rGO}$ 薄膜的光电探测器的 $I-V$ 曲线放大图中 红色曲线位于黑色曲线下方, 即 $I_{\mathrm{ph}}<0$. 这表明 P-rGO-P 光电探测器的 $I-V$ 曲线随着还原温度的增加, 光电探测 器的 $I_{\mathrm{ph}}$ 值也与没有 PDMS 封装的自支撑 $\mathrm{rGO}$ 光电探测 器一样, 从正值 $\left(100,200,300\right.$ 和 $\left.800{ }^{\circ} \mathrm{C}\right)$ 变为负值 $(1000$ 和 $\left.1200{ }^{\circ} \mathrm{C}\right)$, 未封装的 $\mathrm{rGO}$ 光电探测器测试结果中, $I_{\mathrm{ph}}$ 值从基于 $300{ }^{\circ} \mathrm{C}$ 热还原 $\mathrm{rGO}$ 薄膜制备的器件起变为负 值, 与之不同的是, P-rGO-P 光电探测器的测试结果中, $I_{\mathrm{ph}}$ 值从基于 $1000{ }^{\circ} \mathrm{C}$ 热还原 $\mathrm{rGO}$ 薄膜制备的器件起变为 负值.

图 11 为 P-rGO-P 光电探测器的电流-时间 $(I-t)$ 曲线 (红外激光波长: $1064 \mathrm{~nm}$, 功率: $64.8 \mathrm{~mW}$, 偏置电压: $0.5 \mathrm{~V})$. 在 $I-t$ 曲线中, 对光响应电流的光照电流 $\left(I_{\text {light }}\right)$ 和 暗黑电流 $\left(I_{\mathrm{dark}}\right)$ 取差值, 记为 $I_{\mathrm{ph}}$, 代表光电探测器的光电 流.

由图可知，与没有 $\mathrm{PDMS}$ 封装的自支撑 $\mathrm{rGO}$ 光电探 测器的测试结果相比: (1)基于 $100{ }^{\circ} \mathrm{C}$ 热还原所得 $\mathrm{rGO}$ 薄膜制备的 P-rGO-P 光电探测器的响应信号(图 11a)更 加微弱; (2)其它 P-rGO-P 柔性光电探测器在光电快门开 关的过程中均有明显的电流变化响应及与未封装的 $\mathrm{rGO}$ 光电探测器相似的 $I-t$ 曲线变化趋势; (3) 随着 $\mathrm{rGO}$ 热还原温度的增加, P-rGO-P 光电探测器的 $I_{\mathrm{ph}}$ 也表现出 了与自支撑 $\mathrm{rGO}$ 光电探测器相似的变化规律. 基于 100 , 

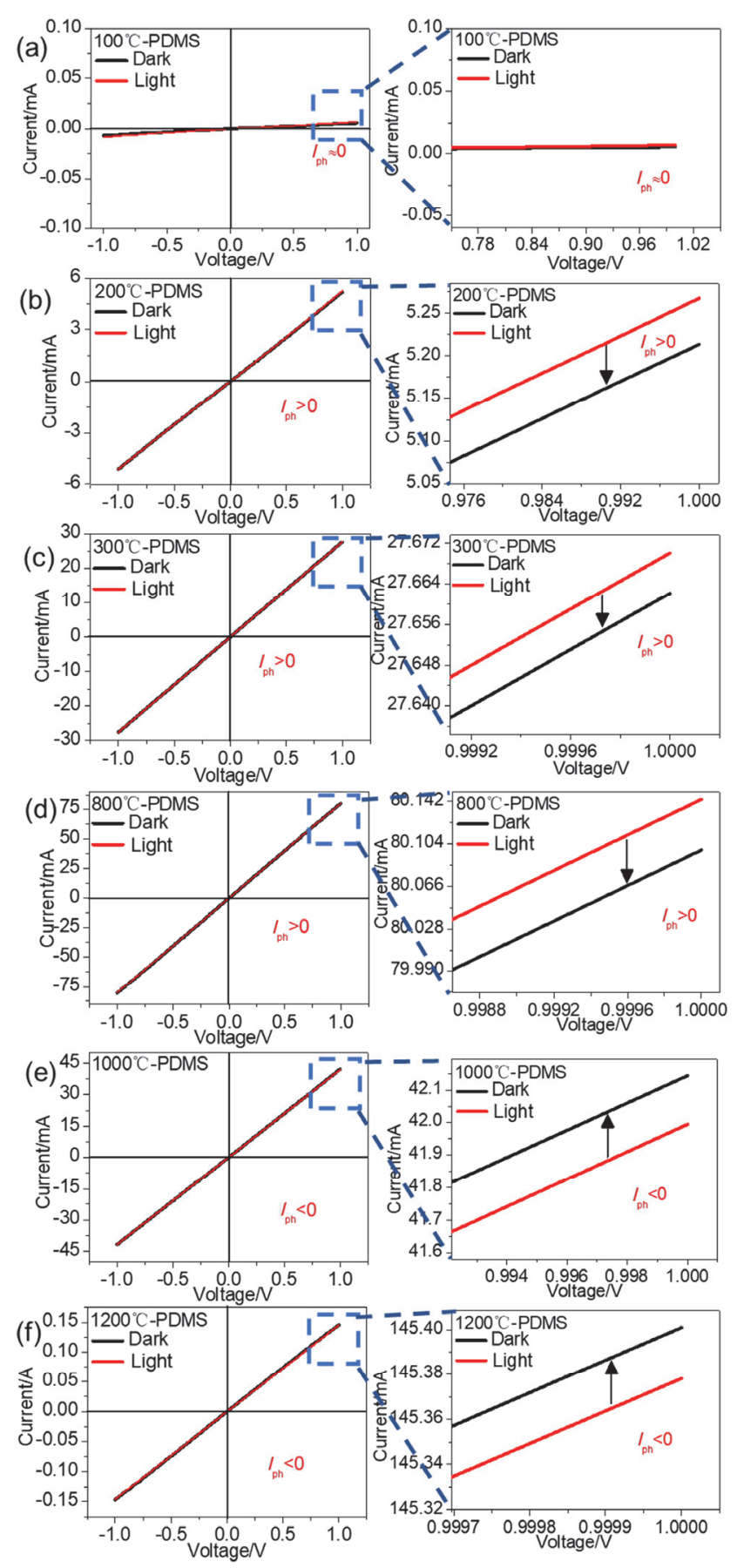

图 10 分别在无光照和 $1064 \mathrm{~nm}$ 激光照射下测试的 $I-V$ 曲线, 测试基 于 $100,200,300,800,1000,1200{ }^{\circ} \mathrm{C}$ 下热还原 $\mathrm{rGO}$ 薄膜制备的 P-rGO-P 光电探测器(红外激光波长: $1064 \mathrm{~nm}$, 功率: $64.8 \mathrm{~mW}$, 偏置电 压: $-1 \sim 1 \mathrm{~V}$ )

Figure $10 I-V$ curves measured in dark and upon $1064 \mathrm{~nm}$ laser illumination, respectively, of P-rGO-P detectors based on rGO thin films annealed at 100, 200,300, 800, 1000, $1200{ }^{\circ} \mathrm{C}$ (laser wavelength: $1064 \mathrm{~nm}$, power: $64.8 \mathrm{~mW}$, bias voltage: $-1 \sim 1 \mathrm{~V}$ )

200,300 和 $800{ }^{\circ} \mathrm{C}$ 热还原的 rGO 薄膜所制备的 P-rGO-P 光电探测器, 当光电快门打开, 有激光照射在叉指电极 上时 $(\mathrm{ON})$, 其电流会快速增加并达到平衡, $I_{\mathrm{ph}}>0$. 而基 于 1000 和 $1200{ }^{\circ} \mathrm{C}$ 热还原的 rGO 薄膜所制备的 P-rGO-P
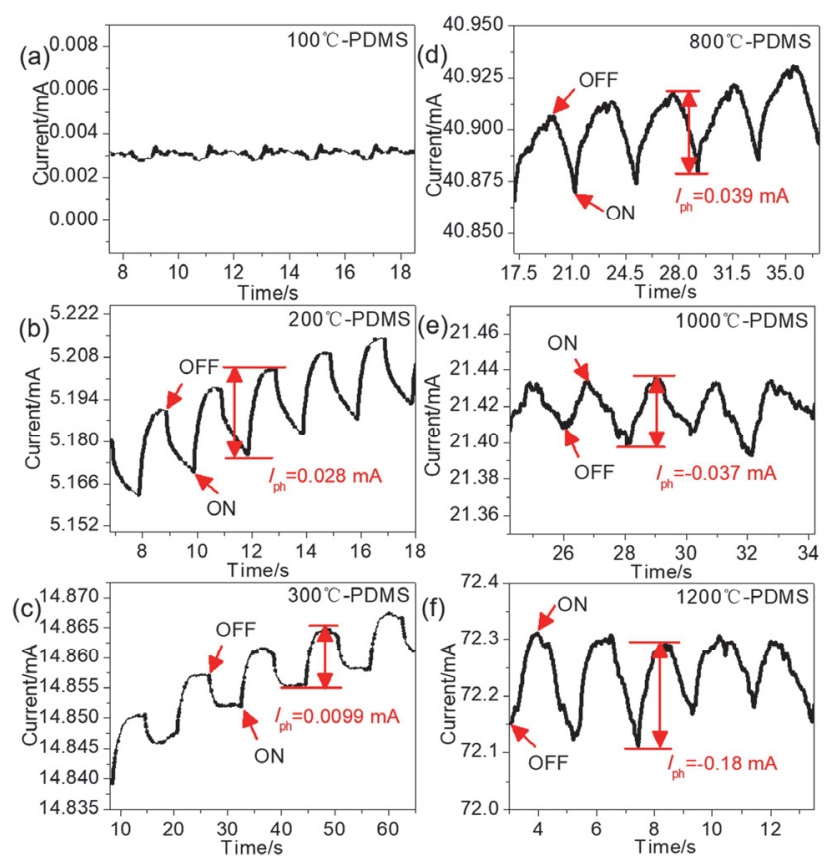

图 11 由 $100,200,300,800,1000,1200{ }^{\circ} \mathrm{C}$ 下还原所得 $\mathrm{rGO}$ 制备的 P-rGO-P 光电探测器的电流-时间曲线(红外激光波长: $1064 \mathrm{~nm}$, 功率: $64.8 \mathrm{~mW}$, 偏置电压: $0.5 \mathrm{~V}$ )

Figure 11 I- $t$ curves of P-rGO-P detectors based on rGO thin films annealed at 100, 200, 300, 800,1000, $1200{ }^{\circ} \mathrm{C}$, respectively (laser wavelength: $1064 \mathrm{~nm}$, power: $64.8 \mathrm{~mW}$, bias voltage: $0.5 \mathrm{~V}$ )

光电探测器, 当光电快门打开, 有激光照射在叉指电极 上时，电流会快速减小并达到平衡, $I_{\mathrm{ph}}<0$. 但未封装的 $\mathrm{rGO}$ 光电探测器从基于 $300{ }^{\circ} \mathrm{C}$ 热还原所得 $\mathrm{rGO}$ 薄膜制 备的光电探测器起, 测试电流就会在有激光照射在叉指 电极上时, 快速减小并达到平衡, 而 P-rGO-P 光电探测 器从基于 $1000{ }^{\circ} \mathrm{C}$ 热还原所得 $\mathrm{rGO}$ 薄膜的光电探测器 起, 测试电流才会在有激光照射在叉指电极上时, 快速 减小并达到平衡; (4) P-rGO-P 光电探测器的 $I_{\mathrm{ph}}$ 数值小 于相应的没有 PDMS 封装光电探测器的 $I_{\mathrm{ph}}$ 数值.

导致 P-rGO-P 柔性光电探测器的响应率与未封装 $\mathrm{rGO}$ 光电探测器响应率存在差异这一现象的主要原因 是, 封装材料 PDMS 本身在 $1064 \mathrm{~nm}$ 波长处有一定吸收 (见 SI, 图 S2), 但 PDMS 并没有明显的光电效应(见 SI, 图 S3), 且 PDMS 本身不导电 ${ }^{[31]}$, 无法传导光电流, 不 会与 $\mathrm{rGO}$ 形成异质结进而影响光响应电流, 但 PDMS 作为祄底与 $r G O$ 薄膜接触可能引起缓慢的热传递, 从而 消耗部分入射光能量 ${ }^{[32]}$. 另一方面, 用来封装的 PDMS 层可能对金电极产生光诱导表面等离子极化子的过程 有影响, 使其负光电导率降低, 从而令 P-rGO-P 光电探 测器在使用还原温度更高, 还原效果更好的 $\mathrm{rGO}$ 薄膜时 才出现 $I_{\mathrm{ph}}<0$ 的现象. 此外, 在热还原所得的 $\mathrm{rGO}$ 表面 上仍然有含氧官能团残余, 这些官能团与 PDMS 表面上 的基团相互作用，可能会影响光生载流子的寿命、载流 子的迁移速率以及 $\mathrm{rGO}$ 的电阻率，降低了 $\mathrm{rGO}$ 具有的正 
光响应性, 从而最终降低了 P-rGO-P 柔性光电探测器的 光响应 $I_{\mathrm{ph}}$ 值.

表 1 为用在各个还原温度下所得 $\mathrm{rGO}$ 薄膜制备的 光电探测器件的光响应率. 由表可知, 除基于 $100{ }^{\circ} \mathrm{C}$ 热 还原 rGO 薄膜制备所得 P-rGO-P 柔性光电探测器的响 应信号微弱外, 其余光电探测器均对红外光有良好的光 电响应, 且响应率数值大部分低于未封装的光电探测 器. 但对基于 $800{ }^{\circ} \mathrm{C}$ 热还原 $\mathrm{rGO}$ 薄膜所制备的光电探 测器, PDMS 封装后的光电测器的响应率略微高于未封 装的光电探测器, 这可能是受 PDMS 封装层影响的金电 极的负光电导率和 rGO 薄膜的正光响应性共同作用的 结果. 此外, 相比于其它温度, 由 $1200{ }^{\circ} \mathrm{C}$ 下还原所得 的 rGO 薄膜制备的 P-rGO-P 光电探测器光响应率最高, 达到了 $2.78 \mathrm{~mA} / \mathrm{W}$. P-rGO-P 柔性光电探测器具有多种 潜在的实际应用前景. 如图 $12 \mathrm{a}$ 所示, 我们利用 P-rGO-P 光电探测器进行了人体红外辐射探测. 图 $12 \mathrm{a}$ 中的 COVER 代表将食指遮盖在器件的叉指电极上方, UNCOVER 代表将食指从电极上方移开, 我们对每一个 器件都在相同的环境条件进行了反复的试验. 图 $12 \mathrm{~b} \sim$ $12 \mathrm{~g}$ 表明, 当手指移开和贴近时探测器所输出的电流信 号都有明显的、呈规律性的与手指的贴近和远离相对应 的信号变化. 受环境辐射产生的背景电流影响, 响应电 流最低值与最高值的差值 $(\Delta I)$ 与红外激光响应测试结果 不同, 数值始终为正值, 且均可达 $\mathrm{mA}$ 量级, 其中基于 $1200{ }^{\circ} \mathrm{C}$ 热还原 $\mathrm{rGO}$ 薄膜制备的 P-rGO-P 柔性光电探测 器对于人体红外辐射的响应信号可达 $26.2 \mathrm{~mA}(\Delta I)$. 为 了排除自然光对测试的影响, 我们对 P-rGO-P 柔性光电 探测器在有无自然光下进行了测试, 如支撑材料中图 4 所示. 测试结果表明, 对基于 $1200{ }^{\circ} \mathrm{C}$ 热还原所得 $\mathrm{rGO}$ 薄膜制备的 P-rGO-P 光电探测器来说, 遮挡自然光引起 的光响应电流变化的最大值仅为 $1.4 \mathrm{~mA}$, 远小于上述 该器件的光响应电流变化值 $(26.2 \mathrm{~mA})$. 因为遮挡自然 光会导致光响应电流向正方向移动(见 SI, 图 S4), 所以
因人体红外辐射引起的实际电流变化值会略高于 26.2 $\mathrm{mA}$. 因此, P-rGO-P 器件对人体发出的红外辐射有非常 灵敏的响应，可实现对人体红外辐射的探测.

表 1 在 $0.5 \mathrm{~V}$ 偏置电压和激光照射 $(1064 \mathrm{~nm})$ 下, 基于各个温度热还 原所得 $\mathrm{rGO}$ 薄膜制备的光电探测器件的光响应率

Table 1 Responsivities at a bias voltage of $0.5 \mathrm{~V}$ under laser illumination $(1064 \mathrm{~nm})$ of the detectors based on rGO films annealed at varied temperatures

\begin{tabular}{ccccccc}
\hline \multicolumn{7}{c}{ Responsivity at $1064 \mathrm{~nm}(\mathrm{~mA} / \mathrm{W})$} \\
\hline Sample & $100{ }^{\circ} \mathrm{C}$ & $200{ }^{\circ} \mathrm{C}$ & $300{ }^{\circ} \mathrm{C}$ & $800{ }^{\circ} \mathrm{C}$ & $1000{ }^{\circ} \mathrm{C}$ & $1200{ }^{\circ} \mathrm{C}$ \\
P-rGO-P & - & 0.43 & 0.15 & 0.60 & 0.57 & 2.78 \\
no PDMS & 0.00093 & 3.73 & 2.43 & 0.478 & 19.94 & 18.31 \\
\hline
\end{tabular}

此外, 基于 P-rGO-P 光电探测器优秀的柔性和 $\mathrm{rGO}$ 本身的层状堆叠结构(图 7), 我们进一步对基于 $1200{ }^{\circ} \mathrm{C}$ 热还原 $\mathrm{rGO}$ 薄膜制备的器件进行了一系列压感测试, 如 图 13a 所示. 由图 13b 可知, 在机械弯曲测试中, 相比未 封装的 rGO 器件, P-rGO-P 柔性器件极大地增强了柔性, 即使反复弯曲折叠至 $90^{\circ}$ 或 $180^{\circ}, \mathrm{PDMS}$ 封装的 $\mathrm{rGO}$ 薄 膜与电路均不会受到破坏, 器件仍能保持良好的完整 性，在测试过程中可以得到稳定、清晰的电信号，且其 响应信号 $\Delta I$ (电流的最大值与最小值差值)与弯曲程度对 应, 当弯曲 $90^{\circ}$ 时, $\Delta I$ 为 $1.55 \mathrm{~mA}$, 弯曲为 $180^{\circ}$ 时, $\Delta I$ 为 $1.67 \mathrm{~mA}$.

我们也尝试基于 $1200{ }^{\circ} \mathrm{C}$ 热还原 $\mathrm{rGO}$ 薄膜制备的 P-rGO-P 柔性器件测试了人体脉搏响应(图 14a). 图 14b 中黑线为未接触人体皮肤时的测试曲线, 绿线为器件接 触皮肤后的测试曲线，对比二者可知绿线中的信号源于 人体心跳搏动. 我们对所有原始信号的上下峰值计算差 值, 并计算其平均值得出 $\Delta I(0.059 \mathrm{~mA})$. 绿色原始信号 中的大峰对应于心脏的收缩期, 伴随的小峰对应于心脏 的舒张期. 考虑到观测心跳间隔时选取收缩期的大峰更 为直观, 我们去除了原始数据中 $\Delta I$ 远小于 $0.059 \mathrm{~mA}$ 的 峰(也就是舒张期杂乱的小峰), 得到蓝色虚线. 由蓝色 虚线计算得到的心脏收缩时间间隔(即相邻峰值的时间
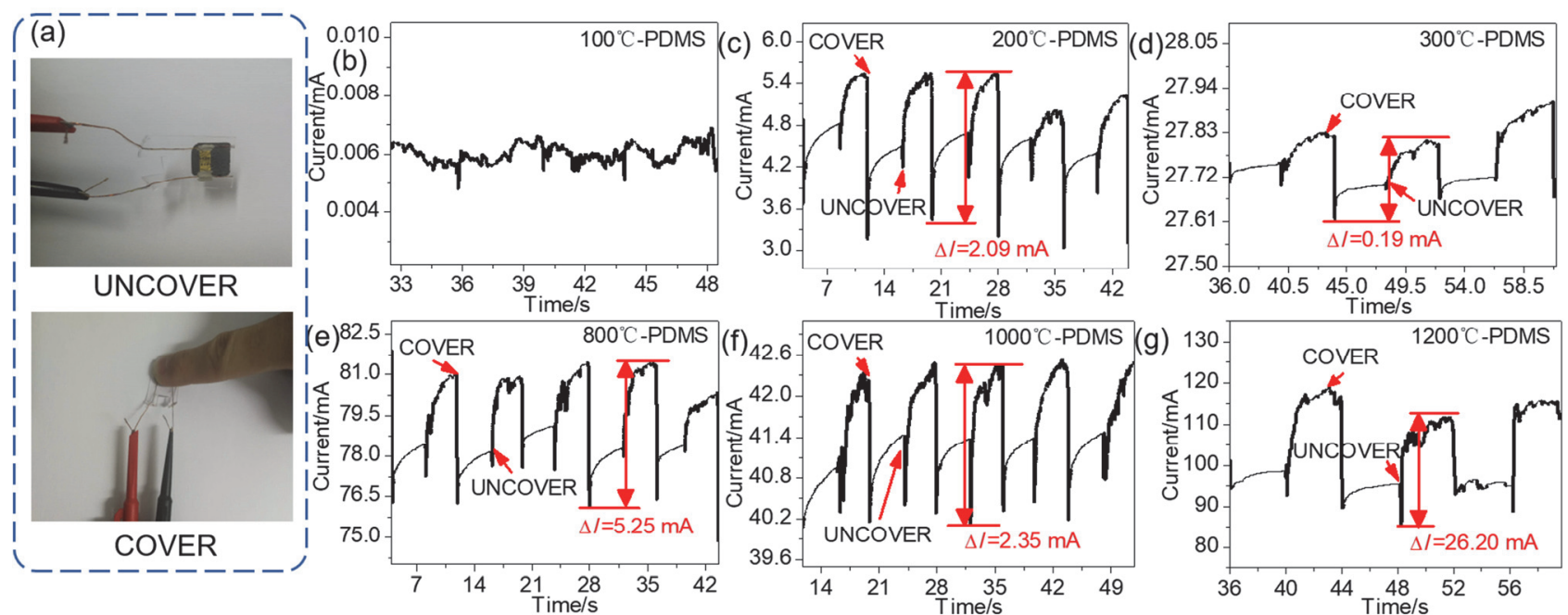

图 12 P-rGO-P 光电探测器对人体红外辐射测试的 $(a)$ 示意图; $(\mathrm{b} \sim \mathrm{g})$ 电流-时间响应曲线(偏置电压: $1 \mathrm{~V}$ )

Figure 12 (a) Schematic diagram of the P-rGO-P detector for human infrared radiation test; $(\mathrm{b} \sim \mathrm{g}) I-t$ curves of tests (bias voltage: $1 \mathrm{~V}$ ) 

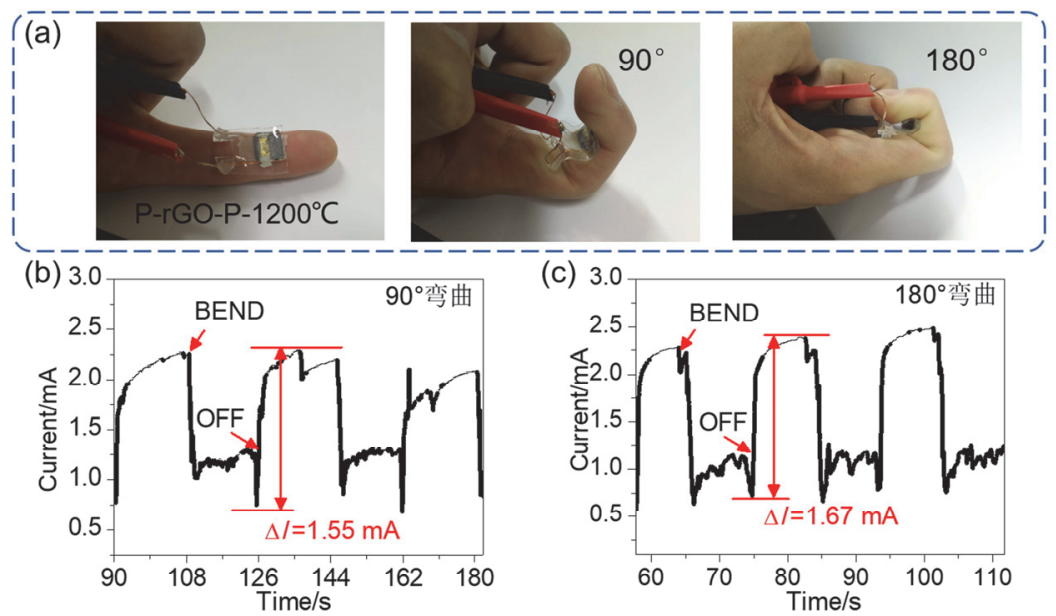

图 13 P-rGO-P 探测器(基于 $1200{ }^{\circ} \mathrm{C}$ 热还原 $\mathrm{rGO}$ 薄膜制备)机械弯曲测试时的(a)示意图; (b)电流-时间曲线(反复弯曲角度为 $90^{\circ}$ ); (c)电流-时间曲 线(反复弯曲角度为 $180^{\circ}$ )(偏置电压: $1 \mathrm{~V}$ )

Figure 13 (a) Schematic diagram of the P-rGO-P detector (based on rGO film annealed at $1200{ }^{\circ} \mathrm{C}$ ) for mechanical bending test (bias voltage: $1 \mathrm{~V}$ ); (b) $I-t$ curves of test (repeated bending angle: $90^{\circ}$ ); (c) $I-t$ curves of test (repeated bending angle: $180^{\circ}$ )

(a)
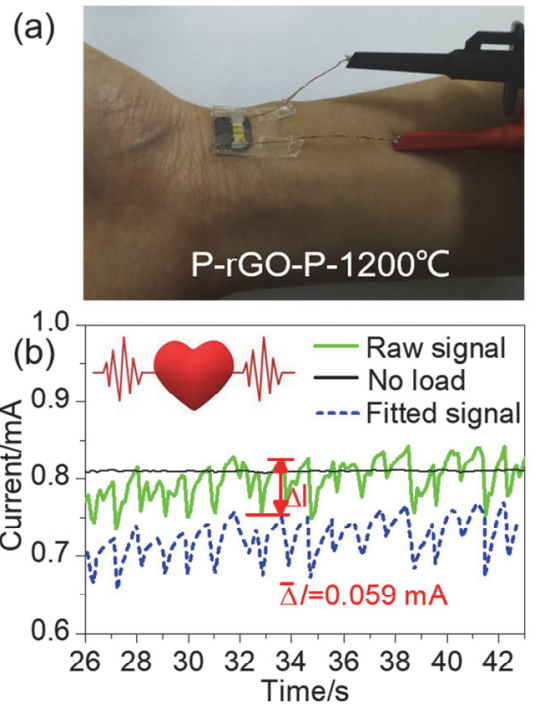

图 14 P-rGO-P 探测器(基于 $1200{ }^{\circ} \mathrm{C}$ 热还原 $\mathrm{rGO}$ 薄膜制备)对人体心 跳测试的(a)示意图, (b)电流-时间响应曲线(偏置电压: $1 \mathrm{~V}$ )

Figure 14 (a) Schematic diagram of the P-rGO-P detector (based on rGO film annealed at $1200{ }^{\circ} \mathrm{C}$ ) for human heartbeat test, (b) $I-t$ curves of test (bias voltage: $1 \mathrm{~V}$ )

间隔)平均值为 $0.946 \mathrm{~s}$, 也就是 63 次/分钟, 完全符合被 测人心跳搏动速度(每分钟 $60 \sim 70$ 次).

P-rGO-P 光电探测器之所以能拥有优秀的压力感应 性能，一方面可归因于我们所使用 $\mathrm{rGO}$ 薄膜是通过逐层 滴涂 $\mathrm{rGO}$ /乙醇分散液来制备的, 经过高温热还原, $\mathrm{rGO}$ 薄膜在微观上仍保持着由 $\mathrm{rGO}$ 薄层堆叠出的多层结构, 这种结构使得 P-rGO-P 柔性器件在受到弯折和压力时, 片层间距被压缩, 原本的接触面积发生变化, 使电阻随 之改变, 进而转变为电信号输出. 另一方面, PDMS 封 装层没有和 $\mathrm{rGO}$ 薄膜层完全紧密地贴合，而是留有一定
的空隙, 两侧的空隙保证了器件在受到弯曲和挤压时, $\mathrm{rGO}$ 薄膜不会因为 PDMS 的粘附而受到过多的应力，拥 有足够的缓冲空间来保持薄膜的完整性. 这两方面都可 以从 P-rGO-P 柔性器件的 SEM 截面示意图中清楚地观 察到(图 7d).

\section{3 结论}

本文基于宽光谱响应的还原氧化石墨烯基自支撑 柔性光电探测器, 通过简单、高效的 PDMS 封装法, 成 功制备了 P-rGO-P 柔性红外探测器. 其厚度只有 800 $\mu \mathrm{m}$, 非常轻便, 并且具有非常好的柔性, 反复弯曲折叠 $180^{\circ}$ 后不影响其性能. P-rGO-P 柔性红外探测器在 1064 $\mathrm{nm}$ 波段激光照射下，响应率最高可达 $2.78 \mathrm{~mA} / \mathrm{W}$, 其对 人体红外辐射响应速度快且响应率高, 能达到 $\mathrm{mA}$ 量级. 此外, P-rGO-P 柔性红外探测器也具有优秀的压敏性. 这种三明治结构的封装模式能够有效地大幅度提升光 电探测材料的柔性，基于该结构的 P-rGO-P 柔性红外探 测器为发展可穿戴柔性多功能器件提供了新的设计思 路, 在生物监测、航空航天、智能电子等领域具有潜在 的应用前景.

\section{4 实验部分}

\section{$4.1 \mathrm{GO}$ 分散液的制备}

按照改进 Hummers 方法 ${ }^{[33]}$ 制备 GO 分散液: 取 3.6 $\mathrm{g}$ 石墨、 $6.0 \mathrm{~g} \mathrm{~K}_{2} \mathrm{~S}_{2} \mathrm{O}_{8} 、 6.2 \mathrm{~g}$ 白磷和 $28.8 \mathrm{~mL}$ 浓硫酸于圆 底烧瓶中混合, 混合物在 $80{ }^{\circ} \mathrm{C}$ 油浴中摚拌 $4.5 \mathrm{~h}$. 用去 离子水反复洗涤混合物，抽滤，放入 $60{ }^{\circ} \mathrm{C}$ 烘箱中干燥 $12 \mathrm{~h}$.

取 $2 \mathrm{~g} \mathrm{NaNO}_{3}$ 和 $92 \mathrm{~mL}$ 浓硫酸与干燥样品于圆底烧 瓶中混合, 冰浴中搅拌 $1 \mathrm{~h}$. 搅拌过程中, 将 $12 \mathrm{~g}$ 
$\mathrm{KMnO}_{4}$ 少量多次加入到悬浮液中, 期间保持混合物温 度低于室温. 移出冰浴, 将混合物在水浴中升温至 $35{ }^{\circ} \mathrm{C}$, 搅拌 $2 \mathrm{~h}$. 缓慢加入 $184 \mathrm{~mL}$ 去离子水, 搅拌 $2 \mathrm{~h}$. 向充分摚拌的县浮液中加入 $160 \mathrm{~mL}$ 去离子水和 $25 \mathrm{~mL}$ $\mathrm{H}_{2} \mathrm{O}_{2}$, 搅拌 $30 \mathrm{~min}$ 后用质量分数 $5 \% \mathrm{HCl}$ 和去离子水多 次离心 $(5000 \mathrm{r} / \mathrm{min})$ 洗涤, 将所得产物冷冻干燥 $24 \mathrm{~h}$, 得 到 GO.

取 $355.5 \mathrm{mg} \mathrm{GO}$ 于烧杯中, 加入 $71.1 \mathrm{~mL}$ 乙醇, 超 声分散配制 $5 \mathrm{mg} / \mathrm{mL}$ 的 $\mathrm{GO} /$ 乙醇分散液, 稀释后得到 $0.7 \mathrm{mg} / \mathrm{mL}$ 的 $\mathrm{GO} /$ 乙醇分散液.

\section{$4.2 \mathrm{rGO}$ 薄膜的制备}

将 $0.7 \mathrm{mg} / \mathrm{mL}$ 的 $\mathrm{GO} /$ 乙醇分散液按照 $75 \mu \mathrm{L} / \mathrm{cm}^{2}$ 的 用量滴涂在刻蚀的硅纳米线上, 每滴涂完一次, 入 $60{ }^{\circ} \mathrm{C}$ 烘箱干燥 $5 \mathrm{~min}$ 后再进行下一次滴涂, 滴涂 6 次后, 将样品置于室温下干燥 $12 \mathrm{~h}$.

干燥后的样品通过管式炉分别于 $100,200,300$, 800, 1000, $1200{ }^{\circ} \mathrm{C}$ 下进行还原 $\left[95 \% \mathrm{Ar}-5 \% \mathrm{H}_{2}\right.$ (体积比)], 热处理 $3 \mathrm{~h}$ 后, 最终得到 6 种不同还原温度下的 $\mathrm{rGO}$ 薄 膜.

\subsection{P-rGO-P 柔性器件的制备}

将 $\mathrm{rGO}$ 薄膜在高真空电阻蒸发镀膜设备中镀上 $\mathrm{Au}$ 叉指电极后备用.

在直径 $3.5 \mathrm{~cm}$ 的培养血中滴加 $400 \mu \mathrm{L}$ PDMS 以及 $40 \mu \mathrm{L}$ 固化剂 KH550 (PDMS 和固化剂的体积比为 10 : 1), 入 $50{ }^{\circ} \mathrm{C}$ 烘箱干燥 $2 \mathrm{~h}$. 将已镀上 $\mathrm{Au}$ 叉指电极的 $\mathrm{rGO}$ 薄膜粘在 PDMS 上, 用银胶作为胶粘剂粘接 $\mathrm{Au}$ 叉指电 极与 $\mathrm{Cu}$ 导线. 干燥后, 旋涂 $(500 \mathrm{r} / \mathrm{min}) 600 \mu \mathrm{L}$ PDMS 对器件进行封装, 之后放入 $50{ }^{\circ} \mathrm{C}$ 烘箱干燥一晚, 得到 P-rGO-P 柔性器件.

\section{References}

[1] Hong, G.; Diao, S.; Antaris, A. L.; Dai, H. Chem. Rev. 2015, 115, 10816.

[2] Ko, H. C.; Stoykovich, M. P.; Song, J.; Malyarchuk, V.; Choi, W. M.; Yu, C.-J.; Geddes III, J. B.; Xiao, J.; Wang, S.; Huang, Y.; Rogers, J. A. Nature 2008, 454, 748.

[3] Martyniuk, P.; Rogalski, A. Prog. Quantum Electron. 2008, 32, 89.

[4] Rauch, T.; Böberl, M.; Tedde, S. F.; Fürst, J.; Kovalenko, M. V.; Hesser, G.; Lemmer, U.; Heiss, W.; Hayden, O. Nat. Photonics 2009, 3,332 .

[5] Rogalski, A.; Chrzanowski, K. Metrol. Meas. Syst. 2014, 21, 565.
[6] Lv, J. T.; Yang, L. J. ; Li, Z. G.; Wei, Y. T.; Zhang, B. J.; Liang, L. Q.; Wang, F. W.; Si, G. Y. Acta Chim. Sinica 2013, 71, 1275. (吕江 涛, 杨琳娟, 李志刚, 魏永涛, 张宝健, 梁丽勤, 王凤文, 司光远, 化学学报, 2013, 71, 1275.)

[7] Zhou, J. P.; Wu, B. G.; Zhou, Z. K.; Tian, J. W.; Yuan, A. H. Chin. J. Org. Chem. 2019, 39, 406. (周建平, 吴保庚, 周志宽, 田蒋为, 袁 爱华, 有机化学, 2019, 39, 406.)

[8] Juang, F.-S.; Su, Y.-K.; Yu, H. H.; Liu, K.-J. Mater. Chem. Phys. 2003, 78, 620 .

[9] Xie, C.; Yan, F. Small 2017, 13, UNSP 1701822.

[10] Geim, A. K.; Novoselov, K. S. Nat. Mater. 2007, 6, 183.

[11] Wu, H.-Q.; Linghu, C.-Y.; Lu, H.-M.; Qian, H. Chin. Phys. B 2013, 22, 098106.

[12] Zhu, J.; Yang, X.; Fu, Z.; He, J.; Wang, C.; Wu, W.; Zhang, L. Chemistry 2016, 22, 2515.

[13] Gong, M.; Liu, Q.; Cook, B.; Kattel, B.; Wang, T.; Chan, W. L.; Ewing, D.; Casper, M.; Stramel, A.; Wu, J. Z. ACS Nano 2017, 11, 4114.

[14] Haider, G.; Roy, P.; Chiang, C.-W.; Tan, W.-C.; Liou, Y.-R.; Chang, H.-T.; Liang, C.-T.; Shih, W.-H.; Chen, Y.-F. Adv. Funct. Mater. 2016, 26, 620 .

[15] Manga, K. K.; Wang, J.; Lin, M.; Zhang, J.; Nesladek, M.; Nalla, V.; Ji, W.; Loh, K. P. Adv. Mater. 2012, 24, 1697.

[16] Dang, V. Q.; Han, G.-S.; Trung, T. Q.; Duy, L. T.; Jin, Y.-U.; Hwang, B.-U.; Jung, H.-S.; Lee, N.-E. Carbon 2016, 105, 353.

[17] De Fazio, D.; Goykhman, I.; Yoon, D.; Bruna, M.; Eiden, A.; Milana, S.; Sassi, U.; Barbone, M.; Dumcenco, D.; Marinov, K.; Kis, A.; Ferrari, A. C. ACS Nano 2016, 10, 8252.

[18] Xu, H.; Wu, J.; Feng, Q.; Mao, N.; Wang, C.; Zhang, J. Small 2014, $10,2300$.

[19] Cai, J.; Ruffieux, P.; Jaafar, R.; Bieri, M.; Braun, T.; Blankenburg, S.; Muoth, M.; Seitsonen, A. P.; Saleh, M.; Feng, X.; Müllen, K.; Fasel, R. Nature 2010, 466, 470.

[20] Eda, G.; Mattevi, C.; Yamaguchi, H.; Kim, H.; Chhowalla, M. J. Phys. Chem. C 2009, 113, 15768.

[21] Cao, Y.; Yang, H.; Zhao, Y.; Zhang, Y.; Ren, T.; Jin, B.; He, J.; Sun, J.-L. ACS Photonics 2017, 4, 2797.

[22] Cao, Y.; Zhu, J.; Xu, J.; He, J.; Sun, J. L.; Wang, Y.; Zhao, Z. Small 2014, 10, 2345.

[23] Yang, H.; Cao, Y.; He, J.; Zhang, Y.; Jin, B.; Sun, J.-L.; Wang, Y.; Zhao, Z. Carbon 2017, 115, 561.

[24] Huang, W.; Dong, X.; Cai, Y. Chin. Sci. Bull. 2016, 62, 635.

[25] Yan, S.; Zhang, G.; Jiang, H.; Li, F.; Zhang, L.; Xia, Y.; Wang, Z.; Wu, Y.; Li, H. ACS Appl. Mater. Interfaces 2019, 11, 10736.

[26] Sun, J.-L.; Zhang, W.; Zhu, J.-L.; Bao, Y. Opt. Express 2010, 18, 4066.

[27] Zheng, J.-G.; Sun, J.-L.; Xue, P. Chin. Phys. Lett. 2011, 28, 127302.

[28] Koppens, F. H.; Mueller, T.; Avouris, P.; Ferrari, A. C.; Vitiello, M. S.; Polini, M. Nat. Nanotech. 2014, 9, 780.

[29] Ito, Y.; Zhang, W.; Li, J.; Chang, H.; Liu, P.; Fujita, T.; Tan, Y.; Yan, F.; Chen, M. Adv. Funct. Mater. 2016, 26, 1271.

[30] Bae, J. J.; Yoon, J. H.; Jeong, S.; Moon, B. H.; Han, J. T.; Jeong, H. J.; Lee, G. W.; Hwang, H. R.; Lee, Y. H.; Jeong, S. Y.; Lim, S. C. Nanoscale 2015, 7, 15695.

[31] Jiang, F.; Zheng, X. L.; Chen, L.; Hu, N.; Yang, J.; Liao, Y. J. New Chem. Mater. 2016, 44, 7. (姜帆, 郑小林, 陈礼, 胡宁, 杨军, 廖 彦剑, 化工新型材料, 2016, 44, 7.)

[32] Cao, Y.; Zhao, Y.; Wang, Y.; Zhang, Y.; Wen, J.; Zhao, Z.; Zhu, L. Carbon 2019, 144, 193.

[33] Hummers, W. S.; Offeman, R. E. J. Am. Chem. Soc. 1958, 80, 1339.

(Cheng, B.) 